\title{
Performance Comparison of Space-Time Block Coding for Single and Multicarrier CDMA Systems
}

\author{
César A. Medina
}

Tiago T. V. Vinhoza

Raimundo Sampaio-Neto

\begin{abstract}
Resumo-Este trabalho propõe comparações entre sistemas de múltiplo acceso baseados em CDMA em combinação com o esquema de codificação espaço-temporal de Alamouti. Quatro sistemas diferentes são considerados: CDMA multiportadora com intervalo de guarda tipo prefixo cíclico (MC-CDMA CP), CDMA multiportadora com intervalo de guarda tipo preenchimento de zeros (MC-CDMA ZP), CDMA de portadora única com intervalo de guarda tipo prefixo cíclico (SC-CDMA CP) e CDMA de portadora única com intervalo de guarda tipo preenchimento de zeros (SC-CDMA ZP). As comparações são realizadas com o receptor de mínimo erro quadrático médio (MMSE). Os resultados apresentados são: taxa de erro de bit, resistência ao efeito perto-longe e robustez à carga do sistema. É mostrado através de simulações que os sistemas CDMA multiportadora têm um melhor desempenho que os sistemas CDMA de portadora única, e em termos de taxa de erro de bit, sistemas MC-CDMA ZP com códigos Alamouti apresentam melhor desempenho.
\end{abstract}

Palavras-Chave-Alamouti, CDMA Multiportadora, CDMA de portadora única, codificação espaço-temporal.

Abstract-This work provides comparisons between CDMAbased multiple access systems in combination with Alamouti's space-time coding scheme. Four different systems are considered: multicarrier CDMA with cyclic prefix guard interval (MCCDMA CP), multicarrier CDMA with zero padding guard interval (MC-CDMA ZP), single carrier CDMA with cyclic prefix guard interval (SC-CDMA CP) and single carrier CDMA with zero padding guard interval (SC-CDMA ZP). Comparisons are carried out with the minimum mean square error (MMSE). Bit error rate (BER), near-far resistance (NFR) effect and robustness to system load results are show. It is shown through computer simulations that multicarrier CDMA-based systems performs better that single carrier CDMA-based systems, and in terms of bit error rate, MC-CDMA ZP with Alamouti's space-time codes performs better than the others.

Keywords-Alamouti, Multicarrier CDMA, Single-Carrier CDMA, Space-time coding.

\section{INTRODUCTION}

Future wireless communication systems have to support a wide range of multimedia services such as speech, image, and data transmission with different and variable bit rates, QoS and latency. These requirements cannot be entirely covered by the second generation systems, primarily designed for speech transmissions. An important challenge for fourth generation systems is the selection of an appropriate multiple access scheme which provides the data transmission at rates of

César A. Medina, Tiago T. V. Vinhoza e Raimundo Sampaio-Neto, Centro de Estudos em Telecomunicações (CETUC), Pontifícia Universidade do Rio de Janeiro (PUC-Rio), Rio de Janeiro (RJ), Brasil, E-mails: \{csmedina, vinhoza, raimundo $\} @$ cetuc.puc-rio.br. Este trabalho foi parcialmente financiado pelo $\mathrm{CNPq}$.
$100 \mathrm{Mb} / \mathrm{s}$ for high-mobility applications to $1 \mathrm{~Gb} / \mathrm{s}$ for lowmobility applications [1] and high spectrum efficiency up to $10 \mathrm{~b} / \mathrm{s} / \mathrm{Hz}$ [2].

In recent years, single carrier (SC) block transmission and OFDM systems have been widely studied in multiple user access schemes, such as CDMA. Single carrier CDMA (SCCDMA) is the well known CDMA with a guard interval between each symbol [3], [4]. The chips are transmitted sequentially over the whole bandwidth allocated for that user. Multicarrier CDMA (MC-CDMA) is based on the concatenation of DS (direct sequence) spreading and OFDM technique. The data symbol of a user is spreaded and the chips are simultaneously transmitted, each one over a narrowband subchannel by the multicarrier modulation (frequency domain spreading). The combination of direct sequence code division multiple access (DS-CDMA) and multi-carrier modulation was first proposed in 1993 [5], [6].

On the other hand, MIMO technology which uses multiple antennas to transmit and receive signals has gained a lot of attention as an effective diversity technique to combat fading and/or increase the capacity of wireless networks [1], [7]. One of such techniques is the Alamouti space-time coding [8] which uses two transmit and multiple receive antennas. Alamouti's code is the only existing complex orthogonal design with full rate, full diversity and minimal delay [7], [9].

The aim of this work is to provide a comparison between these multiple access systems (SC, MC- CDMA) in combination with Alamouti's scheme. Both zero padding (ZP) and cyclic prefix (CP) types of guard intervals are considered. Comparison is carried out with the decision directed minimum mean square error (DD-MMSE) receiver, bit error rate (BER), near-far resistance (NFR) effect and robustness to system load results are show.

This paper is organized as follows: Section II describes a unified framework of a Alamouti block transmission system under which both SC-CDMA and MC-CDMA are described. Section III addresses the Zero-Forcing receiver and the diversity gain of these systems. In Section IV the minimum mean squared error (MMSE) receiver is presented. The decision directed MMSE (DD-MMSE) is introduced in Section V. In Section VI we present the results obtained trough computer simulations. Finally, Section VII gives the conclusions.

\section{Alamouti Block Transmission System}

A discrete model of a block space-time system employing Alamouti's [8] scheme is depicted in Fig. 1. This scheme uses two transmit antennas and one receive antenna, although any 
number of receive antennas could be used with an appropriate combining method such as maximal ratio combining (MRC). Block Alamouti proceeds as follows. The modulated symbols passes through the space-time encoder, $\boldsymbol{X}(i)$, which maps the symbols of user $k$ according to:

$$
\boldsymbol{X}(i)=\sqrt{\rho_{k}}\left[\begin{array}{cc}
s_{k}(2 i) & -s_{k}^{*}(2 i+1) \\
s_{k}(2 i+1) & s_{k}^{*}(2 i)
\end{array}\right]
$$

where $\rho_{k}=A_{k} / 2, A_{k}$ is the power of the transmitted symbol for user $k$, and $(\cdot)^{*}$ denotes complex conjugate. It is assumed that symbols $s_{k}(i)$ are independently and identically distributed (i.i.d.) with zero mean and variance $\sigma_{s}^{2}=1$. Different rows of $\boldsymbol{X}(i)$ refer to different branches of the transmitter (see Fig. 1) whereas different columns refer to different symbol periods.
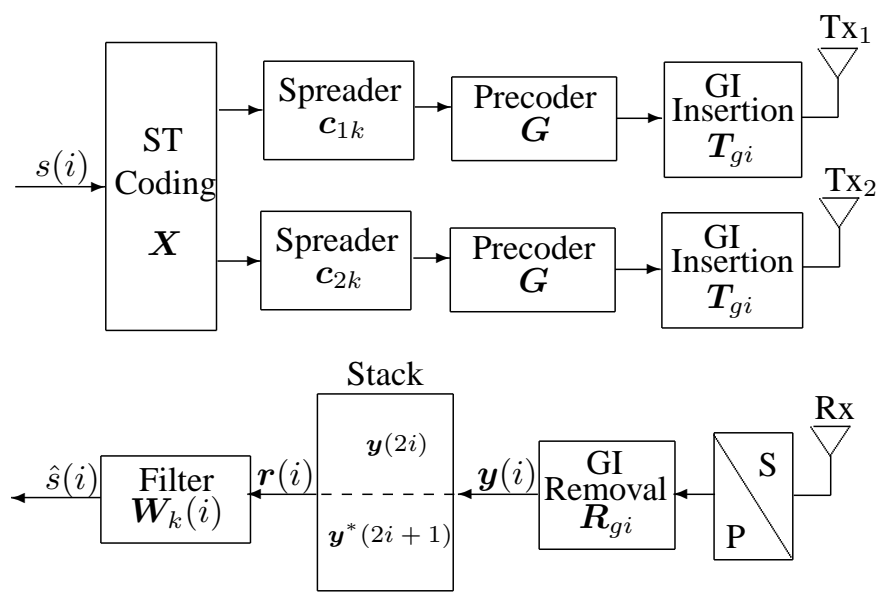

Fig. 1. Alamouti block transmissions system

Two spreading codes of $M$ binary chips per symbol are assigned to each user, one for each row of $\boldsymbol{X}(i)$. The two spreading codes may be formed from a single spreading code $\boldsymbol{c}$ as follows: $\boldsymbol{c}_{1 k}=\left[\boldsymbol{c}^{T}, \mathbf{0}^{T}\right]$ and $\boldsymbol{c}_{2 k}=\left[\mathbf{0}^{T}, \boldsymbol{c}^{T}\right]$, a scheme for UMTS W-CDMA or $\boldsymbol{c}_{1 k}=\left[\boldsymbol{c}^{T}, \boldsymbol{c}^{T}\right]$ and $\boldsymbol{c}_{2 k}=\left[\boldsymbol{c}^{T},-\boldsymbol{c}^{T}\right]$, adopted in the IS-2000 standard [10].

Each spreaded symbol is precoded by the $M \times M$ matrix $G$ that represents an arbitrary linear operation performed before transmission. After that, in order to avoid interblock interference (IBI) at the receiver, a guard interval of length $G$ is inserted, $G$ must be at least the channel order. This operation is represented by a $P \times M$ matrix $\boldsymbol{T}_{g i}$, where $P=M+G$. For the particular cases of zero padding and cyclic prefixing the guard interval insertion is implemented by the matrices $\boldsymbol{T}_{z p}$ and $\boldsymbol{T}_{c p}$ given below.

$$
\boldsymbol{T}_{z p}=\left[\begin{array}{c}
\boldsymbol{I}_{M} \\
\mathbf{0}_{G \times M}
\end{array}\right] \quad \boldsymbol{T}_{c p}=\left[\begin{array}{c}
\mathbf{0}_{G \times M-G} \mid \boldsymbol{I}_{G} \\
\boldsymbol{I}_{M}
\end{array}\right]
$$

where $\boldsymbol{I}_{k}$ represents a $k \times k$ identity matrix and $\mathbf{0}_{m \times n}$ represents an $m \times n$ null matrix. Finally, each block is serialized and transmitted through antenna $T_{x 1}$ or $T_{x 2}$, according to $\boldsymbol{X}(i)$.

The channel impulse response from the $j$-th $(j=1,2)$ transmitter to the receiver is modelled here as a FIR filter with $L$ taps whose gains are samples of the channel impulse response complex envelope.

Assuming that during two symbol periods each multipath channel impulse response remains constant, that is, $\boldsymbol{h}_{j}(2 i)=$ $\boldsymbol{h}_{j}(2 i+1)=\left[h_{j, 0}(2 i) \ldots h_{j, L-1}(2 i)\right]^{T}$, the transmission through the multipath channel can be represented by a $P \times P$ lower triangular Toeplitz convolution matrix $\boldsymbol{H}_{j}(2 i)$, whose first column is $\left[h_{j, 0}(2 i) \ldots h_{j, L-1}(2 i) \quad 0 \ldots 0\right]^{T}$

Assuming downlink scenario, where the users experience the same channel conditions, the received vectors collected over two consecutive symbol periods are

$$
\begin{aligned}
\boldsymbol{y}(2 i)= & \sum_{k=1}^{K} \sqrt{\rho_{k}}\left[\boldsymbol{H}_{1}(2 i) \boldsymbol{T}_{g i} \boldsymbol{G} \boldsymbol{c}_{1 k} s_{k}(2 i)\right. \\
& \left.+\boldsymbol{H}_{2}(2 i) \boldsymbol{T}_{g i} \boldsymbol{G} \boldsymbol{c}_{2 k} s_{k}(2 i+1)\right] \\
& +\boldsymbol{\eta}(2 i)+\boldsymbol{n}(2 i) \\
\boldsymbol{y}(2 i+1)= & \sum_{k=1}^{K} \sqrt{\rho_{k}}\left[-\boldsymbol{H}_{1}(2 i) \boldsymbol{T}_{g i} \boldsymbol{G} \boldsymbol{c}_{1 k} s_{k}^{*}(2 i+1)\right. \\
& \left.+\boldsymbol{H}_{2}(2 i) \boldsymbol{T}_{g i} \boldsymbol{G} \boldsymbol{c}_{2 k} s_{k}^{*}(2 i)\right] \\
& +\boldsymbol{\eta}(2 i+1)+\boldsymbol{n}(2 i+1)
\end{aligned}
$$

where $\boldsymbol{n}(i)$ is a complex white Gaussian noise vector whose covariance matrix E $\left[\boldsymbol{n}(i) \boldsymbol{n}^{H}(i)\right]=\sigma_{n}^{2} \boldsymbol{I}_{P}, \boldsymbol{\eta}(i)$ represents the IBI, and $(\cdot)^{H}$ denote the Hermitian transpose. The operator $\mathrm{E}[\cdot]$ stands for ensemble average.

For block transmission with $\mathrm{CP}$, the receiver must remove the guard interval from the received signal to eliminate IBI $(\overline{\boldsymbol{\eta}}(i)=\mathbf{0})$. In the ZP case, these removal is not necessary. This removal is represented by the matrix $\boldsymbol{R}_{g i}=\boldsymbol{R}_{z p}=\boldsymbol{I}_{P}$ for ZP systems and $\boldsymbol{R}_{g i}=\boldsymbol{R}_{c p}=\left[\mathbf{0}_{M \times G} \mid \boldsymbol{I}_{M}\right]$ for CP systems.

Stacking the column vectors $\boldsymbol{y}(2 i)$ and $\boldsymbol{y}^{*}(2 i+1)$ we get the observation vector

$$
\begin{aligned}
\boldsymbol{r}(i)= & {\left[\begin{array}{c}
\boldsymbol{y}(2 i) \\
\boldsymbol{y}^{*}(2 i+1)
\end{array}\right] } \\
= & \sum_{k=1}^{K} \sqrt{\rho_{k}}\left[\begin{array}{cc}
\overline{\boldsymbol{H}}_{1}(2 i) \boldsymbol{c}_{1 k} & \overline{\boldsymbol{H}}_{2}(2 i) \boldsymbol{c}_{2 k} \\
\overline{\boldsymbol{H}}_{2}^{*}(2 i) \boldsymbol{c}_{2 k} & -\overline{\boldsymbol{H}}_{1}^{*}(2 i) \boldsymbol{c}_{1 k}
\end{array}\right] \\
& \cdot\left[\begin{array}{c}
s_{k}(2 i) \\
s_{k}(2 i+1)
\end{array}\right]+\overline{\boldsymbol{n}}(i)
\end{aligned}
$$

where $\overline{\boldsymbol{H}}_{j}(2 i)=\boldsymbol{R}_{g i} \boldsymbol{H}_{j}(2 i) \boldsymbol{T}_{g i} \boldsymbol{G}$, and

$$
\overline{\boldsymbol{n}}(i)=\left[\begin{array}{c}
\boldsymbol{R}_{g i} \boldsymbol{n}(2 i) \\
\boldsymbol{R}_{g i} \boldsymbol{n}(2 i+1)
\end{array}\right]
$$

As will be detailed below, for all flavors of space-time coded CDMA systems studied in this work, the vector $\overline{\boldsymbol{H}}_{j}(2 i) \boldsymbol{c}_{j k}$ for a synchronous $K$-user system can be written as $\overline{\boldsymbol{H}}_{j}(2 i) \boldsymbol{c}_{j k}=$ $\boldsymbol{C}_{j k} \boldsymbol{h}_{j}(2 i)$ and $\overline{\boldsymbol{H}}_{j}^{*}(2 i) \boldsymbol{c}_{j k}=\boldsymbol{C}_{j k}^{*} \boldsymbol{h}_{j}^{*}(2 i)$ where $\boldsymbol{C}_{j k}$ is a code related matrix for user $k$. Then,

$$
\begin{aligned}
\boldsymbol{r}(i)= & \sum_{k=1}^{K} \sqrt{\rho_{k}}\left[\begin{array}{cc}
\boldsymbol{C}_{1 k} \boldsymbol{h}_{1}(2 i) & \boldsymbol{C}_{2 k} \boldsymbol{h}_{2}(2 i) \\
\boldsymbol{C}_{2 k}^{*} \boldsymbol{h}_{2}^{*}(2 i) & -\boldsymbol{C}_{1 k}^{*} \boldsymbol{h}_{1}^{*}(2 i)
\end{array}\right] \\
& \cdot\left[\begin{array}{c}
s_{k}(2 i) \\
s_{k}(2 i+1)
\end{array}\right]+\overline{\boldsymbol{n}}(i)
\end{aligned}
$$

There are usually four choices for these matrices, leading to four systems whose parameters are summarized in Table 1, where $\boldsymbol{F}$ is a $M \times M$ matrix that implements a $M$-point FFT, normalized such that, $\boldsymbol{F}^{H} \boldsymbol{F}=\boldsymbol{F} \boldsymbol{F}^{H}=\boldsymbol{I}_{M}$. 
TABELA I

BLOCK TRANSMISSION SYSTEM CONSIDERED

\begin{tabular}{l|c|c|c}
\hline Transmission system & $\boldsymbol{G}$ & $\boldsymbol{T}_{g i}$ & $\boldsymbol{R}_{g i}$ \\
\hline SC-CDMA-CP & $\boldsymbol{I}_{M}$ & $\boldsymbol{T}_{c p}$ & $\boldsymbol{R}_{c p}$ \\
SC-CDMA-ZP & $\boldsymbol{I}_{M}$ & $\boldsymbol{T}_{z p}$ & $\boldsymbol{R}_{z p}$ \\
MC-CDMA-CP & $\boldsymbol{F}^{H}$ & $\boldsymbol{T}_{c p}$ & $\boldsymbol{R}_{c p}$ \\
MC-CDMA-ZP & $\boldsymbol{F}^{H}$ & $\boldsymbol{T}_{z p}$ & $\boldsymbol{R}_{z p}$ \\
\hline
\end{tabular}

\section{A. SC-CDMA-ZP}

In this case, $\boldsymbol{C}_{j k}$ is an $P \times L$ Toeplitz matrix containing shifted versions of the spreading sequence $\boldsymbol{c}_{j k}$ padded with $G$ zeros.

\section{B. $S C-C D M A-C P$}

In this case, $\boldsymbol{C}_{j k}$ is an $M \times L$ circulant matrix containing circularly-shifted versions of the spreading sequence $\boldsymbol{c}_{j k}$.

\section{C. $M C-C D M A-Z P$}

As in the SC case, $C_{j k}$ is an $P \times L$ Toeplitz matrix containing shifted versions of the transformed spreading sequence of the $k$ th user, $\boldsymbol{F}^{H} \boldsymbol{c}_{j k}$, padded with $G$ zeros.

\section{D. $M C-C D M A-C P$}

As in the SC case, $C_{j k}$ is an $M \times L$ circulant matrix containing circularly-shifted versions of the $k$ th user spreading sequence transform $\boldsymbol{F}^{H} \boldsymbol{c}_{j k}$.

\section{ZERO-FORCING RECEIVER AND DIVERSITY GAIN}

The observation vector (6) can be more compactly written as

$$
\boldsymbol{r}(i)=\boldsymbol{C H}(i) \boldsymbol{P} \boldsymbol{s}(i)+\overline{\boldsymbol{n}}(i)
$$

where $\boldsymbol{C}=\left[\boldsymbol{C}_{1} \cdots \boldsymbol{C}_{K}\right], \boldsymbol{H}(i)=\boldsymbol{I}_{K} \otimes \boldsymbol{h}(i), \boldsymbol{P}=$ $\operatorname{diag}\left(\sqrt{\rho_{1}}, \sqrt{\rho_{1}}, \cdots, \sqrt{\rho_{K}}, \sqrt{\rho_{K}}\right)$,

$$
\begin{gathered}
\boldsymbol{C}_{k}=\left[\begin{array}{cccc}
\boldsymbol{C}_{1 k} & \mathbf{0} & \mathbf{0} & \boldsymbol{C}_{2 k}^{*} \\
\mathbf{0} & \boldsymbol{C}_{2 k}^{*} & -\boldsymbol{C}_{1 k} & \mathbf{0}
\end{array}\right] \\
\boldsymbol{h}(i)=\left[\begin{array}{cc}
\boldsymbol{h}_{1}(2 i) & \mathbf{0} \\
\boldsymbol{h}_{2}^{*}(2 i) & \mathbf{0} \\
\mathbf{0} & \boldsymbol{h}_{1}^{*}(2 i) \\
\mathbf{0} & \boldsymbol{h}_{2}(2 i)
\end{array}\right] \\
\boldsymbol{s}(i)=\left[\begin{array}{c}
\boldsymbol{s}_{1}(i) \\
\vdots \\
\boldsymbol{s}_{K}(i)
\end{array}\right]
\end{gathered}
$$

$\boldsymbol{s}_{k}(i)=\left[s_{k}(2 i), s_{k}(2 i+1)\right]$, and $\otimes$ represents the Kronecker product. Then the zero-forcing receiver is given by $\boldsymbol{W}_{Z F}=$ $\left(\boldsymbol{C}^{\dagger}\right)^{H} \boldsymbol{H}(i) \boldsymbol{P}^{-1}$ where $\boldsymbol{C}$ is assumed to have full column rank and the signatures of all the users are known, $(\cdot)^{\dagger}$ is the pseudo-inverse matrix. It follows that

$$
\boldsymbol{z}(i)=\boldsymbol{W}_{Z F}^{H} \boldsymbol{r}(i)=\mathcal{H}(i) \boldsymbol{s}(i)+\boldsymbol{P}^{-1} \boldsymbol{H}^{H}(i) \boldsymbol{C}^{\dagger} \overline{\boldsymbol{n}}(i)
$$

where

$$
\begin{aligned}
\mathcal{H}(i) & =\boldsymbol{P}^{-1} \boldsymbol{H}^{H}(i) \boldsymbol{H}(i) \boldsymbol{P} \\
& =\operatorname{diag}(\overbrace{\|\boldsymbol{\vartheta}\|^{2}, \cdots,\|\boldsymbol{\vartheta}\|^{2}}^{2 K})
\end{aligned}
$$

where $\|\boldsymbol{\vartheta}\|^{2}=\left\|\boldsymbol{h}_{1}(2 i)\right\|^{2}+\left\|\boldsymbol{h}_{2}(2 i)\right\|^{2}$. This result is valid for any $\boldsymbol{R}_{g i}, \boldsymbol{T}_{g i}$, and $\boldsymbol{G}$ with the restriction that the guard interval insertion and removal allows a free IBI transmission.

\section{MMSE RECEIVER}

To facilitate the derivation of the MMSE receiver we use the following expression of the observation vector $\boldsymbol{r}(i)$ in (4)

$$
\boldsymbol{r}(i)=\boldsymbol{\Psi}(i) \boldsymbol{P} \boldsymbol{s}(i)+\overline{\boldsymbol{n}}(i)
$$

where

$$
\boldsymbol{\Psi}(i)=\left[\boldsymbol{\Psi}_{1}(i) \cdots \boldsymbol{\Psi}_{K}(i)\right]
$$

and

$$
\boldsymbol{\Psi}_{k}(i)=\left[\begin{array}{cc}
\overline{\boldsymbol{H}}_{1}(2 i) \boldsymbol{c}_{1 k} & \overline{\boldsymbol{H}}_{2}(2 i) \boldsymbol{c}_{2 k} \\
\overline{\boldsymbol{H}}_{2}^{*}(2 i) \boldsymbol{c}_{2 k} & -\overline{\boldsymbol{H}}_{1}^{*}(2 i) \boldsymbol{c}_{1 k}
\end{array}\right]
$$

The MMSE receiver, $\boldsymbol{W}_{M M S E}$, is obtained by minimizing the mean-squared error criterion

$$
\boldsymbol{W}_{M M S E}=\min \arg _{\boldsymbol{W}} \mathrm{E}\left[\left\|\boldsymbol{s}(i)-\boldsymbol{W}^{H} \boldsymbol{r}(i)\right\|^{2}\right]
$$

The solution to (17) is given by [11]

$$
\boldsymbol{W}_{M M S E}=\boldsymbol{R}_{r r}^{-1} \boldsymbol{R}_{r s}
$$

where $\boldsymbol{R}_{r r}=\mathrm{E}\left[\boldsymbol{r}(i) \boldsymbol{r}^{H}(i)\right]$ and $\boldsymbol{R}_{r s}=\mathrm{E}\left[\boldsymbol{r}(i) \boldsymbol{s}^{H}(i)\right]$. Assuming perfect channel knowledge at the $i$-th instant of time, we can use $\boldsymbol{R}_{r s}=\mathrm{E}[\boldsymbol{\Psi}(i)] \boldsymbol{P}$, where it is assumed that the elements of $\boldsymbol{s}(i)$ are independently and identically distributed (i.i.d.) with variance $\sigma_{s}^{2}=1$.

Note that for the solution (18) is necessary the knowledge of the signatures of all of the users, which it is a non-realistic situation for downlink systems. If only the first user is of interest, the MMSE receiver reduces to

$$
\boldsymbol{W}_{1}=\sqrt{\rho_{1}} \boldsymbol{R}_{r r}^{-1} \mathrm{E}\left[\boldsymbol{\Psi}_{1}(i)\right]
$$

which uses only the signatures of the first user.

\section{DECISION DIRECTED MMSE RECEIVER}

The solution to (19) is possible if we know the channel state information (CSI) and the amplitude of the desired user to construct $\sqrt{\rho_{k}} \boldsymbol{\Psi}_{k}(i)$ and estimate $\boldsymbol{R}_{r s_{k}}=\sqrt{\rho_{k}} \mathrm{E}\left[\boldsymbol{\Psi}_{k}(i)\right]$. The CSI can be obtained by blind [12] or training-based methods. Another approach makes use of training symbols to estimate recursively $\boldsymbol{R}_{r s_{k}}$ and then switch to a decision directed scheme, as follows:

In the training phase:

1) Estimate $\boldsymbol{R}_{r s_{1}}(i)$ through

$$
\boldsymbol{R}_{r s_{1}}(i)=\lambda \boldsymbol{R}_{r_{s_{1}}}(i-1)+(1-\lambda) \boldsymbol{r}(i) \boldsymbol{s}_{1}^{H}(i)
$$

2) Estimate $\boldsymbol{R}_{r r}(i)$ through

$$
\boldsymbol{R}_{r r}(i)=\lambda \boldsymbol{R}_{r r}(i-1)+(1-\lambda) \boldsymbol{r}(i) \boldsymbol{r}^{H}(i)
$$

3) $\boldsymbol{W}_{1}(i)=\boldsymbol{R}_{r r}^{-1}(i) \boldsymbol{R}_{r s_{1}}(i)$ 
In the decision directed phase:

1) Detection: $\hat{\boldsymbol{s}}(i)=\operatorname{disc}\left\{\boldsymbol{W}_{1}^{H}(i-1) \boldsymbol{r}(i)\right\}$, where the component $z_{j}$ of the vector $\mathbf{z}=\operatorname{disc}\{\mathbf{x}\}$ is the symbol of the signal constellation closer to the component $x_{j}$ of $\mathrm{x}$.

2) Estimate $\boldsymbol{R}_{r s_{1}}(i)$ through

$$
\boldsymbol{R}_{r s_{1}}(i)=\lambda \boldsymbol{R}_{r s_{1}}(i-1)+(1-\lambda) \boldsymbol{r}(i) \hat{\boldsymbol{s}}_{1}^{H}(i)
$$

3) Estimate $\boldsymbol{R}_{r r}(i)$ through

$$
\boldsymbol{R}_{r r}(i)=\lambda \boldsymbol{R}_{r r}(i-1)+(1-\lambda) \boldsymbol{r}(i) \boldsymbol{r}^{H}(i)
$$

4) $\boldsymbol{W}_{1}(i)=\boldsymbol{R}_{r r}^{-1}(i) \boldsymbol{R}_{r s_{1}}(i)$

\section{Simulation Results}

The simulation results presented are for BPSK synchronous MC- and SC- CDMA systems that employ Hadamard sequences of length $M=16$. Because we focus on a downlink scenario, users experience the same channel conditions. All channels assume that $G=3$. It is also assumed here that the channels experienced by different users are statistically independent and identically distributed. For fading channels, the sequence of channel coefficients for each user, $h_{l}(i)=$ $p_{l} \alpha_{l}(i)(l=0,1,2, \ldots, L-1)$ is obtained with Clarke's model [13], where it is assumed that channel order $L-1=G$. This procedure corresponds to the generation of independent sequences of correlated unit power complex Gaussian random variables $\left(\mathrm{E}\left[\left|\alpha_{l}^{2}(i)\right|\right]=1\right)$ with the path weights $p_{l}$ normalized so that $\sum_{l=1}^{L_{\mathrm{p}}}\left|p_{l}\right|^{2}=1$. In this work $p_{0}=0.5957+$ $j 0.0101, p_{1}=-0.3273-j 0.3472, p_{2}=0.2910-j 0.0533$ and $p_{3}=0.1285-j 0.5599$. The results are shown in terms of the normalized Doppler frequency $\left(f_{d} T\right)$, where $f_{d}$ is the Doppler frequency and $T$ is the duration of two blocks. In all the simulations the decision directed MMSE receiver was used with 500 symbols in the training phase and 1500 symbols in the decision directed phase. Results are the average of 1000 ensembles.

In Fig. 2 we assess the desired user BER performance of the analyzed receivers versus $E_{b} / N_{0}$. The system has $K=4$ users. Regarding power distribution, we simulate a severe nearfar scenario where each interferer has a power level $20 \mathrm{~dB}$ above the desired user, that is, near-far ratio $(N F R)$ is equal to $20 \mathrm{~dB}$. The channel is time-varying with $f_{d} T=0.001$.

In Fig. 3 we assess the desired user BER performance of the analyzed receivers versus the number of users, $K$ for a fixed signal-to-noise ratio and $f_{d} T=0.001$. The desired user has $E_{b} / N_{0}=15 \mathrm{~dB}$ and its $N F R=10 \mathrm{~dB}$.

In Fig. 4 we assess the desired user BER performance of the analyzed receivers versus different values of $N F R$. The system has $K=4$ users, the desired user has $E_{b} / N_{0}=15 \mathrm{~dB}$ and $f_{d} T=0.001$.

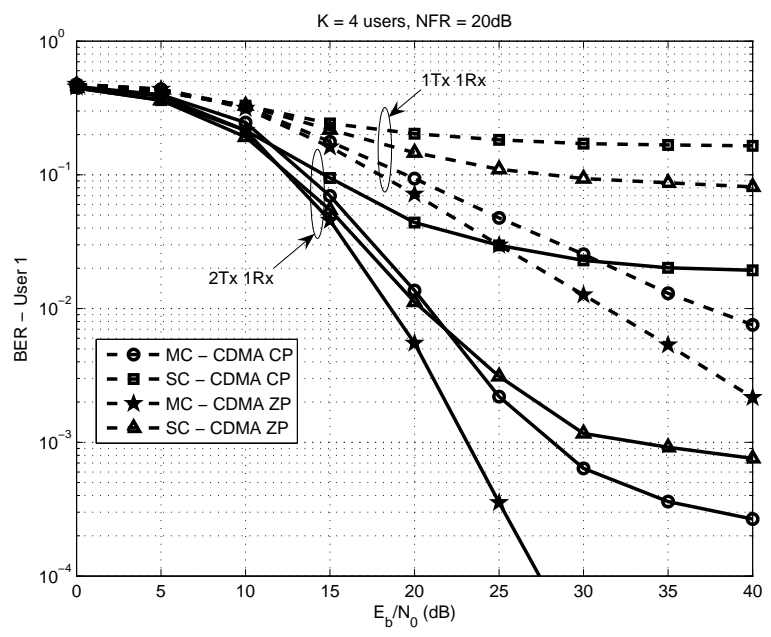

Fig. 2. BER versus $E_{b} / N_{0}(\mathrm{~dB})$

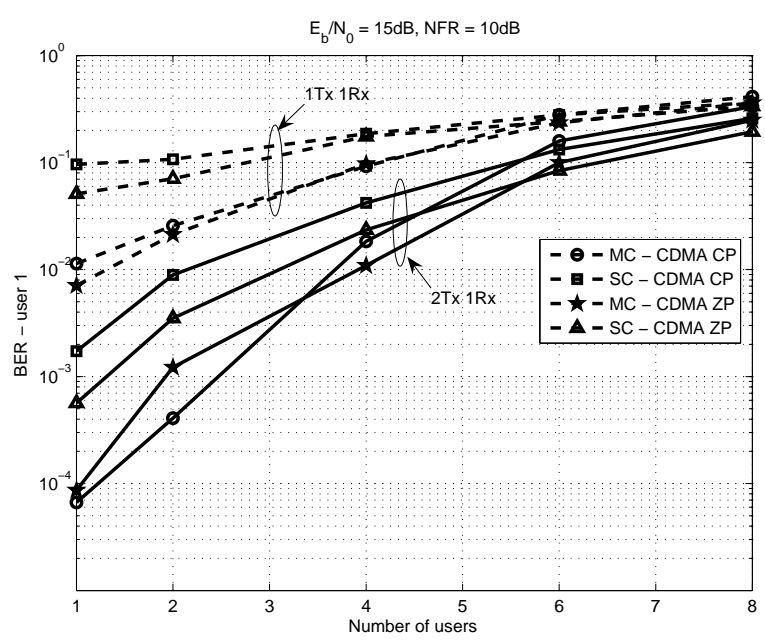

Fig. 3. BER versus Number of users

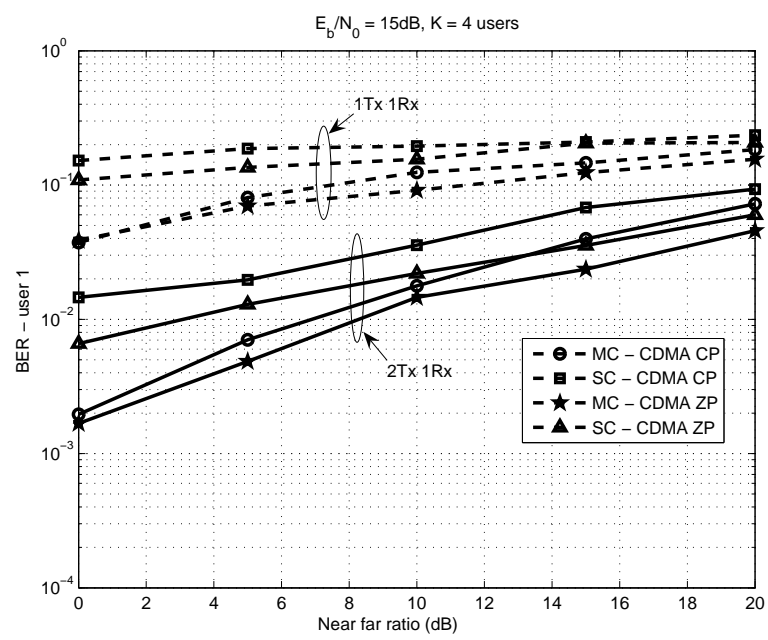

Fig. 4. BER versus $N F R(\mathrm{~dB})$ 
As it can be observed, space-time coded systems show a much lower BER floor than the uncoded systems and up to $17 \mathrm{~dB}$ gain for space-time MC-CDMA CP over uncoded MCCDMA CP for $\mathrm{BER}=10^{-2}$. Also, it is shown that spacetime MC-CDMA systems performs better for low system load, and as the number of users increases space-time SC-CDMA outperforms their multicarrier counterpart. In terms of nearfar resistance, it was observed that space-time MC-CDMA ZP performs slightly better than space-time MC-CDMA CP and that both space-time and uncoded systems are near-far resistant.

\section{CONCLUSIONS}

In this paper we have compared the Alamouti's spacetime coding with single and multicarrier block transmission CDMA-based multiple access systems. The comparison was carried out in terms of bit error rate (BER), near-far resistance (NFR) and robustness to system load, all of these in the decision directed minimum mean squared error receiver. Under the test conditions, it was observed $17 \mathrm{~dB}$ gain for spacetime MC-CDMA CP over uncoded MC-CDMA CP for BER equal to $10^{-2}$. Also, space-time MC-CDMA systems performs better for low system load, and as the number of users increases space-time SC-CDMA outperforms their multicarrier counterpart. In terms of near-far resistance, it was observed that space-time MC-CDMA ZP performs slightly better than space-time MC-CDMA CP.

\section{REFERENCES}

[1] M. Steer, "Beyond 3G," IEEE Microwave Magazine, vol. 8, no. 1, pp. 76-82, February 2007.

[2] H. Yang, "A road to future broadband wireless access; MIMO-OFDM-Based air interface," IEEE Communications Magazine, vol. 43, no. 1, pp. 53-60, January 2005.

[3] K. L. Baum, T. A. Thomas, F. W. Vook, and V. Nangia, "Cyclic-prefix CDMA: an improved transmission method for broadband DS-CDMA cellular systems," Proc. WCNC, vol. 1, pp. 183-188, March 2002.

[4] A. S. Madhukumar, Francois Chin, Ying-Chang Liang, and Kai Yang, "Single-carrier cyclic prefix-assisted CDMA system with frequency domain equalization for high data rate transmission," EURASIP J. Wirel. Commun. Netw., vol. 2004, no. 1, pp. 149-160, 2004.

[5] A. Chouly, A. Brajal, and S. Jourdan, "Orthogonal multicarrier techniques applied to direct sequence spread spectrum CDMA systems," Proc. GLOBECOM, pp. 1723-1728, Nov-Dec. 1993.

[6] V. DaSilva and E. S. Sousa, "Performance of orthogonal CDMA codes for quasi-synchronous communication systems," Proc. ICUPC, pp. 995999, October 1993.

[7] S. Barbarossa, Multiantenna Wireless Communication Systems, Artech House, 2005.

[8] S. M. Alamouti, "A simple transmit diversity technique for wireless communications," IEEE J. Select. Areas Commun., vol. 16, no. 8, pp. 1451-1458, October 1998.

[9] V. Tarokh, H. Jafarkhani, and A. R. Calderbank, "Space time block codes from orthogonal designs," IEEE Transactions on Information Theory, vol. 45, no. 5, pp. 1456-1467, July 1999.

[10] B. Hochwald, T. L. Marzetta, and C. B. Papadias, "A transmitter diversity scheme for wideband CDMA systems based on space-time spreading," IEEE J. Select. Areas Commun., vol. 19, no. 1, pp. 48-60, January 2001.

[11] S. Haykin, Adaptive Filter Theory, Prentice Hall, 2001.

[12] C. A. Medina, T. T. V. Vinhoza, and R. Sampaio-Neto, "Performance comparison of minimum variance single carrier and multicarrier CDMA receivers," Proc. VIII IEEE Signal Processing Advances in Wireless communications (SPAWC), 2007.

[13] T. S. Rappaport, Wireless Communications: Principles and Practice, Prentice Hall PTR, 1996. 